\title{
Effective Lagrangians for physical degrees of freedom in the Randall-Sundrum model
}

\author{
Edward E. Boos ${ }^{a}$, Yuri A. Kubyshin ${ }^{a, b}$, \\ Mikhail N. Smolyakov ${ }^{c}$ and Igor P. Volobuev ${ }^{a}$ \\ ${ }^{a}$ Institute of Nuclear Physics, Moscow State University \\ 119899 Moscow, Russia \\ ${ }^{b}$ Departament MA IV, Universitat Politècnica de Catalunya \\ Mòd. C-3, Campus Nord, Jordi Girona, 1-3 \\ 08034 Barcelona, Spain \\ ${ }^{c}$ Physics Department, Moscow State University \\ 119899 Moscow, Russia
}

October 25, 2018

\begin{abstract}
We derive the second variation Lagrangian of the Randall - Sundrum model with two branes, study its gauge invariance and diagonalize it in the unitary gauge. We also show that the effective fourdimensional theory looks different on different branes and calculate the observable mass spectra and the couplings of the physical degrees of freedom of 5-dimensional gravity to matter.
\end{abstract}

\section{Introduction}

The Randall-Sundrum (RS) model with two branes, first proposed and analyzed in Refs. [1, 2], has been extensively studied in the literature, see Refs. [3] - [5] for reviews. The interest in this model, known as the RS1 model, is 
due to a novel solution of the hierarchy problem suggested within it, and to a possibility to check its predictions in the planned collider experiments (see articles [6, 7] and Refs. [5, 8, 9] for reviews).

The RS model describes gravity propagating in a five-dimensional spacetime with the fifth dimension being compactified to the orbifold $S^{1} / Z_{2}$ with the circumference of the circle equal to $2 R$. Let us denote coordinates of the space-time by $\left\{\hat{x}^{M}\right\}=\left\{\left(x^{\mu}, y\right)\right\}, M=0,1,2,3,4$, so that $\left\{x^{\mu}\right\}, \mu=0,1,2,3$ are the coordinates of the four-dimensional space-time and $\hat{x}^{4} \equiv y$ is the coordinate along the fifth dimension. $Z_{2}$-symmetry of the orbifold is realized by identifying points $\left(x^{\mu}, y\right)$ and $\left(x^{\mu},-y\right)$, where $y$ is a point of the circle. In addition we have the usual periodicity condition which identifies $\left(x^{\mu}, y\right)$ and $\left(x^{\mu}, y+2 R\right)$. The branes are located at the fixed points $y=0$ and $y=R$ of the orbifold.

The action of the RS1 model is equal to

$$
S=S_{g}+S_{1}+S_{2}
$$

where $S_{g}$ is the five-dimensional Einstein action with the cosmological term,

$$
S_{g}=\frac{1}{16 \pi \hat{G}} \int(\mathcal{R}-\Lambda) \sqrt{-g} d^{5} \hat{x}
$$

and $S_{1}$ and $S_{2}$ are the standard actions of the branes:

$$
\begin{aligned}
& S_{1}=V_{1} \int \sqrt{-\tilde{g}} \delta(y) d^{5} \hat{x}, \\
& S_{2}=V_{2} \int \sqrt{-\tilde{g}} \delta(y-R) d^{5} \hat{x} .
\end{aligned}
$$

Here $\hat{G}$ is the five-dimensional gravitational constant, $g_{M N}$ is the metric tensor in five dimensions with the signature $(-,+,+,+,+)$, and $\tilde{g}=\operatorname{det}\left(g_{\mu \nu}\right)$ is the determinant of the induced metric.

The exact background solution for the metric in this model is of nonfactorizable type and is given by [1]

$$
d s^{2}=\gamma_{M N} d \hat{x}^{M} d \hat{x}^{N}=e^{2 \sigma(y)} \eta_{\mu \nu} d x^{\mu} d x^{\nu}+(d y)^{2},
$$

where $\eta_{\mu \nu}$ is the Minkowski metric. The function $\sigma(y)$ is equal to $\sigma(y)=$ $-k|y|$ for $-R \leq y \leq R$ and is continued periodically outside this interval. 
The parameter $k$ has the dimension of mass, and the cosmological constant $\Lambda$ and the brane tensions $V_{1}, V_{2}$ are related to it as follows:

$$
\Lambda=-12 k^{2}, \quad V_{1}=-V_{2}=-\frac{3 k}{4 \pi \hat{G}} .
$$

If $k>0$ the brane at $y=0$ (brane 1 ) has positive tension and the one at $y=R$ (brane 2) has negative tension.

Though many studies of this model have been done already, some issues have not been properly understood and clarified. Thus earlier papers dealt only with the equations of motion for the linearized gravity, and its Lagrangian description remained practically untouched. In the present paper we first repeat the derivation of the Lagrangian of quadratic fluctuations around the background solution (4) and carry out its diagonalization. We also discuss the problem of imposing a gauge condition in a consistent way and introduce the unitary gauge, in which the radion is represented explicitly by a four-dimensional scalar field.

In the majority of papers on the RS1 model masses and couplings constants in effective theories on the branes are calculated with respect to the induced metric. However, as one can see from Eq. (4), the induced metric on brane 2 contains the conformal factor $e^{2 \sigma(R)}=e^{-2 k R}$. Hence the coordinate system $\left\{x^{\mu}\right\}$ is not Galiliean on the brane (coordinates are called Galillean if $g_{\mu \nu}=\operatorname{diag}(-1,1,1,1)$, see for example Ref. [10]) and, therefore, the naive identification of parameters from the Lagrangian leads to wrong conclusions. This issue was recognized and discussed in Ref. [11]. In this paper, a covariant procedure was proposed, which consists in using covariant equations and expressing all coordinate distances in terms of proper distances. For example, within this approach the masses of brane fields, i.e. fields localized on a brane and not propagating in the five-dimensional bulk, were determined from the rate of exponential falloff of the 2-point function. It was shown that such masses measured by brane observers are independent of the warp factor and are given by the Lagrangian masses. In Ref. [11] it was also confirmed that Newton's constant of gravitational interaction between two masses depends on the warp factor in the same way as in the conventional interpretation [12]. Also, masses of bulk scalar fields were analyzed there. It was found that an observer on the negative tension brane sees particles with masses $m_{n} \sim k$, whereas an observer on the positive tension brane detects exponentially suppressed masses $m_{n} \sim k e^{-k R}$. 
In the present paper we re-examine these issues and calculate the physical masses and coupling constants on each of the branes in a more straightforward and, as we believe, simpler way. Instead of analyzing the 2-point function and the geodesic equation we pass to the Galilean coordinates and perform necessary rescalings of fields. This allows us to get the correct identification of the physical parameters in the effective theory on the branes. We calculate the mass spectra and couplings of the Kaluza-Klein modes of the gravitons to matter on the branes, as well as the masses of brane fields.

As it is usual in scenarios considered in the literature, we assume that our brane is brane 2 (the one with negative tension) and our considerations lead to a phenomenology very similar to the one discussed in Refs. $[6,7]$. However, the general physical picture of the brane world appears to be quite different. Thus, in our approach the fundamental physical scale, i.e. the fivedimensional "Plank mass" $M$, should be in the TeV range. It is very natural to have the value for the parameter $k$ in the same TeV range. The fourdimensional Plank scale $M_{P l}$ is the effective scale for the gravitational zero mode interaction on brane 2 . The hierarchy is generated by the exponenential term coming from the warp factor $e^{2 \sigma(y)}$ in Eq. (4). Namely, the Planck mass is related to the fundamental scale by the relation

$$
M_{P l}^{2} \approx \frac{M^{3}}{k} e^{2 k R}
$$

see Refs. [4, 13] and Sect. 4 of the present article. To reproduce the value of $M_{P l}$ the size $R$ has to be chosen such that $k R \approx 30 \div 35$. Therefore, the size of the extra dimension is expected to be about $35 / \mathrm{TeV}^{1}$, much larger than $35 / M_{P l}$ discussed in earlier papers. We will show that for these values of the model parameters the coupling of gravity to matter on the "hidden" brane (brane 1) turns out to be very strong, namely the gravitational constant is proportional to $M^{-1}$ and not to $M_{P l}^{-1}$, as on brane 2 . There are also some differences in the radion interactions which will be discussed below.

The article is organized as follows. In Sect. 2 we review gauge transformations in an arbitrary background and derive an expression for the Lagrangian of quadratic fluctuations which will be convenient for our purposes. In Sect. 3 we use this result for the calculation of the Lagrangian of quadratic fluctuations in the RS1 model, repeat its diagonalization in a systematic way paying

\footnotetext{
${ }^{1}$ As an alternative interpretation this possibility was mentioned in Ref. [1].
} 
attention to the gauge transformations and identify physical degrees of freedom. In parallel we prove the decoupling of the classical equations of motion for these degrees of freedom. In Sect. 4 we study both brane scalar fields and the bulk fields arising from a five-dimensional metric. We calculate their masses and couplings in effective theories on the branes using the Galilean coordinates. Sect. 5 contains some discussion of the results and concluding remarks.

\section{Second variation Lagrangian in a general background}

For the purpose of generality let us consider the standard gravitational action with the cosmological constant in $(4+d)$-dimensional space-time with coordinates $\left\{\hat{x}^{M}\right\}, M=0,1, \cdots d+3$ :

$$
S_{g}=\frac{1}{16 \pi \hat{G}} \int(\mathcal{R}-\Lambda) \sqrt{-g} d^{4+d} \hat{x},
$$

where $\hat{G}$ is the multidimensional gravitational constant, $\mathcal{R}$ is the scalar curvature and $g_{M N}$ is the metric with signature $(-1,1, \cdots 1)$. For $d=1$ this expression coincides with five-dimensional action (1).

Let $\gamma_{M N}$ be a fixed background metric. We denote $\hat{\kappa}=\sqrt{16 \pi \hat{G}}$ and parameterize the metric $g_{M N}$ as

$$
g_{M N}(\hat{x})=\gamma_{M N}(\hat{x})+\hat{\kappa} h_{M N}(\hat{x}) .
$$

If we substitute this formula into (5) and retain only the terms of the zeroth order in $\hat{\kappa}$, we get the following Lagrangian, which is usually called the second variation Lagrangian:

$$
\begin{aligned}
L_{g}^{(2)} / \sqrt{-\gamma} & =-\frac{1}{4}\left(\nabla_{R} h_{M N} \nabla^{R} h^{M N}-\nabla_{R} h \nabla^{R} h+2 \nabla_{M} h^{M N} \nabla_{N} h-\right. \\
& \left.-2 \nabla^{R} h^{M N} \nabla_{M} h_{R N}\right)+\frac{1}{4}(\mathcal{R}-\Lambda)\left(h_{M N} h^{M N}-\frac{1}{2} h h\right)+ \\
& +\mathcal{G}^{M N} h_{M R} h_{N}^{R}-\frac{1}{2} \mathcal{G}^{M N} h_{M N} h,
\end{aligned}
$$


where $\gamma=\operatorname{det} \gamma_{M N}, h=h_{M}^{M}$, and $\mathcal{G}_{M N}=\mathcal{R}_{M N}-\frac{1}{2} \gamma_{M N}(\mathcal{R}-\Lambda)$. The Ricci tensor $\mathcal{R}_{M N}$, the scalar curvature $\mathcal{R}$ and the covariant derivative $\nabla_{M}$ are calculated with respect to the background metric $\gamma_{M N}$. This formula in rather complicated notations can be found in [14].

Action (5) is invariant under general coordinate transformations $\hat{y}^{M}=$ $\hat{y}^{M}(\hat{x})$, the corresponding transformation of the metric being

$$
g_{R S}^{\prime}(\hat{y}) \frac{\partial \hat{y}^{R}}{\partial \hat{x}^{M}} \frac{\partial \hat{y}^{S}}{\partial \hat{x}^{N}}=g_{M N}(\hat{x})
$$

Let us consider infinitesimal coordinate transformations

$$
\hat{y}^{M}(x)=\hat{x}^{M}+\hat{\kappa} \xi^{M}(\hat{x}) .
$$

Representing the initial and transformed metrics as

$$
\begin{aligned}
& g_{M N}(\hat{x})=\gamma_{M N}(\hat{x})+\hat{\kappa} h_{M N}(\hat{x}), \\
& g_{M N}^{\prime}(\hat{y})=\gamma_{M N}(\hat{y})+\hat{\kappa} h_{M N}^{\prime}(\hat{y}),
\end{aligned}
$$

substituting these formulas into Eq. (8), and keeping the terms up to the first order in $\hat{\kappa}$ we arrive at the following transformation law for the variation $h_{M N}$ :

$$
h_{M N}^{\prime}(\hat{x})=h_{M N}(\hat{x})-\left(\nabla_{M} \xi_{N}+\nabla_{N} \xi_{M}\right)
$$

(see, for example, Ref. [15]). It is easy to check that the action built with the second variation Lagrangian (7) is invariant under transformations (9) which, therefore, can be interpreted as gauge transformations of the field $h_{M N}$.

Suppose that the background metric $\gamma_{M N}$ is a solution of the Einstein equations

$$
\mathcal{R}_{M N}-\frac{1}{2} \gamma_{M N}(\mathcal{R}-\Lambda)=8 \pi \hat{G} T_{M N}
$$

with some energy-momentum tensor of the matter $T_{M N}$. Expressing $\mathcal{G}_{M N}$ and $(\mathcal{R}-\Lambda)$ in terms of the energy-momentum tensor $T_{M N}$, substituting it into (7) and transforming the kinetic term to the standard Fierz-Pauli form, we obtain the following second variation Lagrangian

$$
L_{g}^{(2)} / \sqrt{-\gamma}=-\frac{1}{4}\left(\nabla_{R} h_{M N} \nabla^{R} h^{M N}-\nabla_{R} h \nabla^{R} h+2 \nabla_{M} h^{M N} \nabla_{N} h-(10)\right.
$$




$$
\begin{aligned}
& \left.-2 \nabla_{M} h^{M N} \nabla^{R} h_{R N}\right)+\frac{1}{2} h^{M N} h^{P Q} \mathcal{R}_{M P N Q}-\frac{1}{2} h^{M N} h_{N P} \mathcal{R}_{M}^{P}+ \\
& +\frac{\Lambda}{2(d+2)}\left(h_{M N} h^{M N}-\frac{1}{2} h h\right)-\frac{4 \pi \hat{G}}{d+2} T_{R}^{R}\left(h_{M N} h^{M N}-\frac{1}{2} h h\right)+ \\
& +\left(8 \pi \hat{G} T^{M N} h_{M R} h_{N}^{R}-4 \pi \hat{G} T^{M N} h_{M N} h\right),
\end{aligned}
$$

where $\mathcal{R}_{M N P Q}$ and $\mathcal{R}_{M}^{P}$ are the curvature and the Ricci tensors of the metric $\gamma_{M N}$ respectively. In the next section this expression will be used for calculation of the second variation Lagrangian in Randall-Sundrum model.

\section{Second variation Lagrangian in the Randall - Sundrum model}

We write the perturbed metric around the RS solution $\gamma_{M N}$, Eq. (4), in the form given by Eq. (6) with $\hat{\kappa}=M^{-3 / 2}$, where, as in the Introduction, $M$ is the fundamental mass scale ("Planck mass") of the five-dimensional gravity.

In the RS1 model the brane action terms, Eqs. (2), (3), give rise to the energy-momentum tensor equal to

$$
T_{M N}=-\frac{3 k}{4 \pi \hat{G}} \sqrt{\frac{\tilde{\gamma}}{\gamma}} \gamma_{\mu \nu} \delta_{M}^{\mu} \delta_{N}^{\nu}[\delta(y)-\delta(y-R)],
$$

where $\tilde{\gamma}=\operatorname{det}\left(\gamma_{\mu \nu}\right)$. The contribution of the gravitational term $S_{g}$, Eq. (1), is given by formula (10), whereas the contribution of the branes is equal to

$$
\Delta L_{1,2}^{(2)}=3 k\left(h_{\mu \nu} h^{\mu \nu}-\frac{1}{2} \tilde{h} \tilde{h}\right) \sqrt{-\tilde{\gamma}}[\delta(y)-\delta(y-R)],
$$

where $\tilde{h}=\gamma^{\mu \nu} h_{\mu \nu}$. The complete quadratic Lagrangian for the variation $h_{M N}$ can be written as follows:

$$
\begin{aligned}
& L / \sqrt{-\gamma}=-\frac{1}{4}\left(\nabla_{R} h_{M N} \nabla^{R} h^{M N}-\nabla_{R} h \nabla^{R} h+2 \nabla_{M} h^{M N} \nabla_{N} h-\right. \\
& \left.-2 \nabla_{M} h^{M N} \nabla^{R} h_{R N}\right)+\frac{k^{2}}{2}\left(h_{M N} h^{M N}+h h\right)+\left[-2 k h_{M N} h^{M N}+\right. \\
& \left.+k h \tilde{h}-k h_{M \nu} h^{M \nu}+3 k\left(h_{\mu \nu} h^{\mu \nu}-\frac{1}{2} \tilde{h} \tilde{h}\right)\right](\delta(y)-\delta(y-R)) .
\end{aligned}
$$


As in Sect. 2, the covariant derivatives $\nabla_{M}$ are calculated with respect to the background metric $\gamma_{M N}$ of the RS solution and $h=\gamma^{M N} h_{M N}$.

This Lagrangian is invariant under gauge transformations (9). In the case under consideration they can be found explicitly and turn out to be

$$
\begin{aligned}
h_{\mu \nu}^{\prime}(x, y) & =h_{\mu \nu}(x, y)-\left(\partial_{\mu} \xi_{\nu}+\partial_{\nu} \xi_{\mu}+2 \gamma_{\mu \nu} \partial_{4} \sigma \xi_{4}\right) \\
h_{\mu 4}^{\prime}(x, y) & =h_{\mu 4}(x, y)-\left(\partial_{\mu} \xi_{4}+\partial_{4} \xi_{\mu}-2 \partial_{4} \sigma \xi_{\mu}\right) \\
h_{44}^{\prime}(x, y) & =h_{44}(x, y)-2 \partial_{4} \xi_{4},
\end{aligned}
$$

where the functions $\xi^{M}(x, y)$ satisfy the orbifold symmetry conditions

$$
\begin{aligned}
& \xi^{\mu}(x,-y)=\xi^{\mu}(x, y) \\
& \xi^{4}(x,-y)=-\xi^{4}(x, y) .
\end{aligned}
$$

These gauge transformations in other parameterizations were discussed in papers [16] and [17]. We will use them to remove the gauge degrees of freedom of the field $h_{M N}$. To this end we first make a gauge transformation with

$$
\xi_{4}(x, y)=\frac{1}{4} \int_{-y}^{y} h_{44}\left(x, y^{\prime}\right) d y^{\prime}-\frac{x^{4}}{4 R} \int_{-R}^{R} h_{44}\left(x, y^{\prime}\right) d y^{\prime} .
$$

One can easily see that $\xi_{4}$ satisfies the orbifold symmetry condition. After this transformation $h_{44}$ takes the form

$$
h_{44}^{\prime}(x)=\frac{1}{2 R} \int_{-R}^{R} h_{44}\left(x, y^{\prime}\right) d y^{\prime}
$$

and therefore it does not depend on $y$. Moreover, there are no residual gauge transformations involving $\xi_{4}$.

Now let us consider the components $h_{\mu 4}$. Due to the orbifold symmetry

$$
h_{\mu 4}(x,-y)=-h_{\mu 4}(x, y),
$$

the gauge transformations for $h_{\mu 4}$ read

$$
h_{\mu 4}^{\prime}(x, y)=h_{\mu 4}(x, y)-\left(\partial_{4} \xi_{\mu}-2 \partial_{4} \sigma \xi_{\mu}\right) .
$$

The condition $h_{\mu 4}=0$ is imposed by performing gauge transformations (12) with $\xi_{4}=0$ and $\xi_{\mu}$ satisfying

$$
\xi_{\mu}(x, y)=e^{2 \sigma(y)} \int_{0}^{y} e^{-2 \sigma\left(y^{\prime}\right)} h_{\mu 4}\left(x, y^{\prime}\right) d y^{\prime} .
$$


Note that it satisfies orbifold symmetry condition (13). Thus we have imposed the gauge

$$
h_{\mu 4}=0, h_{44}=\phi\left(x^{\mu}\right),
$$

i.e. $h_{44}$ is a function of four-dimensional coordinates only. We will call it the unitary gauge. There still remain gauge transformations satisfying

$$
\partial_{4}\left(e^{-2 \sigma} \xi_{\mu}\right)=0
$$

They will be important for removing the gauge degrees of freedom of the massless mode of the gravitational field.

Varying Lagrangian (11) we get the equations of motion for the fluctuations $h_{M N}(x)$ which, in the unitary gauge, reduce to the following equations for the fields $h_{\mu \nu}(x, y)$ and $\phi(x)$ :

$$
\begin{aligned}
& \frac{1}{2}\left(\partial_{\rho} \partial^{\rho} h_{\mu \nu}-\partial_{\mu} \partial^{\rho} h_{\rho \nu}-\partial_{\nu} \partial^{\rho} h_{\rho \mu}+h_{\mu \nu}^{\prime \prime}\right)-2 k^{2} h_{\mu \nu}+\frac{1}{2} \partial_{\mu} \partial_{\nu} \tilde{h}+\frac{1}{2} \partial_{\mu} \partial_{\nu} \phi \\
& +\frac{1}{2} \gamma_{\mu \nu}\left(\partial^{\rho} \partial^{\sigma} h_{\rho \sigma}-\partial_{\rho} \partial^{\rho} \tilde{h}-\tilde{h}^{\prime \prime}-4 \sigma^{\prime} \tilde{h}^{\prime}-\partial_{\rho} \partial^{\rho} \phi+12 k^{2} \phi\right) \\
& +\left[2 k h_{\mu \nu}-3 k \gamma_{\mu \nu} \phi\right](\delta(y)-\delta(y-R))=0 \\
& \left(\partial_{\mu} \tilde{h}-\partial^{\nu} h_{\mu \nu}\right)^{\prime}-3 \sigma^{\prime} \partial_{\mu} \phi=0 \\
& \frac{1}{2}\left(\partial^{\mu} \partial^{\nu} h_{\mu \nu}-\partial_{\mu} \partial^{\mu} \tilde{h}\right)-\frac{3}{2} \sigma^{\prime} \tilde{h}^{\prime}+6 k^{2} \phi=0
\end{aligned}
$$

where the prime denotes the derivative w.r.t. $y$. In the chosen gauge Eq. (16) is a constraint. Multiplying Eq. (17) by 2 and subtracting it from Eq. (15) contracted with $\gamma^{\mu \nu}$ we obtain an auxiliary equation

$$
\tilde{h}^{\prime \prime}+2 \sigma^{\prime} \tilde{h}^{\prime}-8 k^{2} \phi+\partial_{\mu} \partial^{\mu} \phi+8 k \phi[\delta(y)-\delta(y-R)]=0,
$$

which will be used later.

When written in terms of $h_{\mu \nu}(x, y)$ and $\phi(x)$ the second variation Lagrangian (11) is not diagonal, correspondingly the equations of motion, Eqs. (15) - (17), are coupled. To diagonalize the Lagrangian and to decouple the equations we write the multidimensional gravitational field as

$$
\begin{aligned}
h_{\mu \nu}(x, y) & =b_{\mu \nu}(x, y)+\gamma_{\mu \nu}(y)(\sigma(y)-c) \phi(x) \\
& +\frac{1}{2 k^{2}}\left[\sigma(y)-c+\frac{1}{2}\right] \partial_{\mu} \partial_{\nu} \phi(x)+\frac{c}{4 k^{2}} e^{-2 \sigma(y)} \partial_{\mu} \partial_{\nu} \phi(x),
\end{aligned}
$$


where $c$ is a constant which will be fixed shortly. We will see that the field $b_{\mu \nu}(x, y)$ describes the massless graviton $[1,2]$ and massive Kaluza-Klein spin2 fields, whereas $\phi(x)$ describes a scalar field called the radion. Apparently, the radion as a massless particle was first identified in Ref. [18] (see also [19]) and studied in articles [20,16]. Actually, substitution (19) is suggested by the form of the gauge transformation which transforms the theory from the local Gaussian normal coordinates (i.e. coordinates corresponding to $g_{44}=1$, $\left.g_{\mu 4}=0[16]\right)$, for which the second variation Lagrangian is diagonal in the bulk by construction, to our coordinates. Evidently, just a gauge transformation cannot diagonalize the Lagrangian, because it is gauge invariant. To carry out the diagonalization and to decouple the equations the last term in Eq. (19), the one $\propto e^{-2 \sigma}$, is needed.

It is easy to check that even with arbitrary $c$ Eqs. (15) - (17) decouple in the bulk, i.e. for $0<y<R$. The junction conditions, coming from Eq. (15), are

$$
\begin{aligned}
& b_{\mu \nu}^{\prime}\left(x, y_{i}\right)-\frac{c}{2 k^{2}} \sigma^{\prime}\left(y_{i}\right) e^{-2 \sigma\left(y_{i}\right)} \partial_{\mu} \partial_{\nu} \phi(x) \\
& +\epsilon_{i}\left[2 k b_{\mu \nu}\left(x, y_{i}\right)+\left(\frac{c}{2 k} e^{-2 \sigma\left(y_{i}\right)}+\frac{\sigma\left(y_{i}\right)-c}{k}\right) \partial_{\mu} \partial_{\nu} \phi(x)\right]=0,
\end{aligned}
$$

where $i=1,2, y_{1}=0, y_{2}=R, \epsilon_{1}=+1, \epsilon_{2}=-1$. At $y=0$ the terms $\propto \partial_{\mu} \partial_{\nu} \phi$ vanish for arbitrary $c$. To make them vanish at $y=R$ we choose

$$
c=\frac{k R}{e^{2 k R}-1} \text {. }
$$

Substituting expression (19) into Eq. (18) we get the equality

$$
\left(e^{2 \sigma} \tilde{b}^{\prime}\right)^{\prime}=-2 c \partial_{\mu} \partial^{\mu} \phi
$$

Making the Fourier expansion in $y$ on the orbifold and taking into account that the l.h.s. does not contain the zero mode, we arrive at the conclusion that the radion field satisfies the equation

$$
\partial_{\mu} \partial^{\mu} \phi=0
$$

and $e^{2 \sigma}\left(e^{-2 \sigma} b\right)^{\prime}$ is a function of $x$ only. Applying the same argument the latter implies that $\left(e^{-2 \sigma} b\right)^{\prime}=0$ (see details in Ref. [13]). By performing transformations which satisfy (14) one can impose the gauge

$$
\tilde{b}=b=0 .
$$


It is easy to see that there still remain gauge transformations parameterized by $\xi_{\mu}=e^{2 \sigma} \zeta_{\mu}(x)$ with $\zeta_{\mu}(x)$ satisfying $\partial^{\mu} \zeta_{\mu}=0$. Substituting expression (19) into Eqs. (16), (17) and passing to gauge (22), we arrive at the following relations: $\partial^{\mu} \partial^{\nu} b_{\mu \nu}=0$ and $\left(e^{-2 \sigma} \partial^{\mu} b_{\mu \nu}\right)^{\prime}=0$. The remaining gauge transformations are sufficient to impose the condition

$$
\partial^{\mu} b_{\mu \nu}=0 .
$$

Conditions (22) and (23) define the gauge often called the transverse-traceless (TT) gauge. Having imposed this gauge, we are still left with residual gauge transformations

$$
\xi_{\mu}=e^{2 \sigma} \zeta_{\mu}(x), \quad \partial_{\rho} \partial^{\rho} \zeta_{\mu}=0, \quad \partial^{\mu} \zeta_{\mu}=0,
$$

which, as we will see shortly, are important for determining the number of degrees of freedom of the massless mode of $b_{\mu \nu}$.

In this gauge Eqs. (16), (17) are trivially satisfied. Eq. (15) becomes

$$
\frac{1}{2} \partial_{\rho} \partial^{\rho} b_{\mu \nu}+\frac{1}{2} b_{\mu \nu}^{\prime \prime}-2 k^{2} b_{\mu \nu}+2 k b_{\mu \nu}(\delta(y)-\delta(y-R))=0 .
$$

Thus, we have decoupled the equations of motion.

Now let us consider the second variation action of the theory. Substituting (19) with $c$ given by (20) into Lagrangian (11) and taking into account the TT gauge conditions $(22),(23)$ for $b_{\mu \nu}$, we get

$$
\begin{aligned}
L / \sqrt{-\gamma} & =-\frac{1}{4} \partial_{\rho} b_{\mu \nu} \partial^{\rho} b^{\mu \nu}-\frac{1}{4}\left(\partial_{4} b_{\mu \nu}-2 \partial_{4} \sigma b_{\mu \nu}\right)\left(\partial_{4} b^{\mu \nu}+2 \partial_{4} \sigma b^{\mu \nu}\right) \\
& -\frac{3}{4} c e^{-2 \sigma} \partial_{\mu} \phi \partial^{\mu} \phi .
\end{aligned}
$$

Integrating over $y$ we obtain the following four-dimensional effective Lagrangian for the field $\phi$ :

$$
L_{\phi}=-\frac{3}{4} c \int_{-R}^{R} e^{-2 \sigma(y)} \sqrt{-\gamma} \partial_{\mu} \phi \partial^{\mu} \phi d y=-\frac{3}{2} \frac{k R^{2}}{e^{2 k R}-1} \eta^{\mu \nu} \partial_{\mu} \phi \partial^{\nu} \phi .
$$

To bring the kinetic term to the canonical form we rescale the field according to

$$
\phi(x)=\sqrt{\frac{e^{2 k R}-1}{3 k R^{2}}} \varphi(x) .
$$


It remains to decompose the field $b_{\mu \nu}$ into Kaluza-Klein modes with definite masses and to integrate Eq. (25) over y. Following Refs. $[2,6,7]$ we write

$$
b_{\mu \nu}(x, y)=\sum_{n=0}^{\infty} b_{\mu \nu}^{(n)}(x) \psi_{n}(y),
$$

where $\left\{\psi_{n}(y), \quad n=0,1,2, \ldots\right\}$ form the complete orthogonal system of eigenfunctions of the equation

$$
\left[\frac{1}{2} \frac{d^{2}}{d y^{2}}+2 k(\delta(y)-\delta(y-R))-2 k^{2}\right] \psi_{n}(y)=-\frac{m_{n}^{2}}{2} e^{2 k|y|} \psi_{n}(y)
$$

satisfying the normalization condition

$$
\int_{-R}^{R} e^{2 k|y|} \psi_{m}(y) \psi_{n}(y) d y=\delta_{m n} .
$$

The solutions to the eigenvalue problem are equal to

$$
\psi_{0}(y)=N_{0} e^{-2 k|y|}, \quad \psi_{n}(y)=N_{n} \Psi_{n}(y) \quad(n=1,2, \ldots),
$$

where

$$
\Psi_{n}(y)=C_{1} J_{2}\left(\beta_{n} e^{-(\sigma(y)+k R)}\right)+C_{2} Y_{2}\left(\beta_{n} e^{-(\sigma(y)+k R)}\right)
$$

and $J_{2}(\xi)$ and $Y_{2}(\xi)$ are the Bessel and Neumann functions respectively. The coefficients $C_{1}, C_{2}$ and the values of $\beta_{n}$ are determined by the junction conditions at $y=0$ and $y=R$. One gets that

$$
C_{1}=Y_{1}\left(\beta_{n} e^{-k R}\right), \quad C_{2}=-J_{1}\left(\beta_{n} e^{-k R}\right)
$$

and $\beta_{n}$ are roots of the equation

$$
J_{1}\left(\beta_{n} e^{-k R}\right) Y_{1}\left(\beta_{n}\right)-J_{1}\left(\beta_{n}\right) Y_{1}\left(\beta_{n} e^{-k R}\right)=0 .
$$

As we will see shortly, $e^{-k R} \sim 10^{-15}$ so that for small $n$ the numbers $\beta_{n}$ satisfy approximately the equation $J_{1}\left(\beta_{n}\right)=0$. The values of the first few $\beta_{n}$ are given by $\beta_{n}=3.83,7.02,10.17,13.32$ for $n=1,2,3,4$ respectively. The normalization constants in Eq. (28) are found to be

$$
N_{0}=\frac{\sqrt{k}}{\sqrt{1-e^{-2 k R}}}, \quad N_{n}=\frac{\sqrt{k}}{\sqrt{e^{2 k R} \Psi_{n}^{2}\left(\beta_{n}\right)-\Psi_{n}^{2}\left(\beta_{n} e^{-k R}\right)}} .
$$


For small $n$ the values of functions (28) at $y=0$ and $y=R$ can be estimated as

$$
\begin{aligned}
& \psi_{0}(0)=N_{0} \approx \sqrt{k} ; \quad \psi_{n}(0) \approx \frac{\sqrt{k}}{\left|J_{2}\left(\beta_{n}\right)\right|} e^{-k R}, \quad(n \neq 0) \\
& \psi_{0}(R)=N_{0} e^{-2 k R} \approx \sqrt{k} e^{-2 k R} ; \quad \psi_{n}(R) \approx-\sqrt{k} e^{-k R} \quad(n \neq 0) .
\end{aligned}
$$

Performing mode expansion (27) we obtain the following expression for the four-dimensional effective action (i.e. dimensionally reduced action) of the five-dimensional gravitational field in the RS model:

$$
\begin{aligned}
S_{\text {eff }} & =\int\left[-\frac{1}{4} \sum_{n=0}^{\infty}\left(\partial_{\mu} b_{\rho \sigma}^{(n)} \partial_{\nu} b_{\epsilon \delta}^{(n)} \eta^{\mu \nu} \eta^{\rho \epsilon} \eta^{\sigma \delta}+m_{n}^{2} b_{\mu \rho}^{(n)} b_{\nu \sigma}^{(n)} \eta^{\mu \nu} \eta^{\rho \sigma}\right)\right. \\
& \left.-\frac{1}{2} \eta^{\mu \nu} \partial_{\mu} \varphi \partial_{\nu} \varphi\right] d x
\end{aligned}
$$

where $m_{n}=\beta_{n} k e^{-k R}$. We see that the effective theory includes a massless spin-2 tensor field (the graviton), an infinite tower of massive spin-2 tensor fields (often referred to as massive KK gravitons) and a massless scalar field (the radion) whose classical equation of motion is Eq. (21). An important point is that, due to the form of the zero mode function (see Eq. (28)), remaining gauge freedom (24) allows transformations

$$
b_{\mu \nu}^{(0)}(x) \rightarrow b_{\mu \nu}^{\prime 0}(x)=b_{\mu \nu}^{0}(x)-\left(\partial_{\mu} \zeta_{\nu}(x)+\partial_{\nu} \zeta_{\mu}(x)\right)
$$

of the field $b_{\mu \nu}^{(0)}(x)$ with $\zeta_{\mu}(x)$ satisfying $\partial^{\mu} \zeta_{\mu}(x)=0, \partial_{\mu} \partial^{\mu} \zeta_{\nu}(x)=0$. This guarantees that the field $b_{\mu \nu}^{(0)}(x)$ has only two degrees of freedom and, therefore, can indeed be identified with the field of the massless graviton.

It turns out that the five-dimensional gravity, described by effective action (32), looks different, when viewed from different branes. One of the ways to see this property is to consider the interaction of the five-dimensional gravity with matter on the branes. This problem will be addressed in the next section.

\section{Effective theories on the branes}

To begin with let us consider a scalar field $\Phi(x)$ localized on brane $i(i=1,2)$. Suppose that its dynamics is determined by the standard action with quartic 
coupling

$$
S_{\Phi, i}=-\int_{B_{i}} d x \sqrt{-\gamma\left(x, y_{i}\right)}\left[\frac{1}{2} \gamma^{\mu \nu}\left(x, y_{i}\right) \partial_{\mu} \Phi \partial_{\nu} \Phi+\frac{m^{2}}{2} \Phi^{2}+\frac{\lambda}{4} \Phi^{4}\right],
$$

where, as before, $i=1,2$ and $y_{1}=0, y_{2}=R$. Here we consider the leading approximation in $\hat{\kappa}$, therefore fluctuations $h_{M N}$ (see Eq. (6)) are neglected. The induced metric on brane $i$ is equal to

$$
\begin{aligned}
\gamma_{\mu \nu} & =e^{2 \sigma_{i}} \eta_{\mu \nu}, \\
\sigma_{1} & \equiv \sigma(0)=0, \quad \sigma_{2} \equiv \sigma(R)=-k R .
\end{aligned}
$$

Action (33) can be rewritten as

$$
S_{\Phi, i}=-\int_{B_{i}} d x e^{4 \sigma_{i}}\left[\frac{1}{2} e^{-2 \sigma_{i}} \eta^{\mu \nu} \partial_{\mu} \Phi \partial_{\nu} \Phi+\frac{m^{2}}{2} \Phi^{2}+\frac{\lambda}{4} \Phi^{4}\right] .
$$

The conventional interpretation is obtained by rescaling the field

$$
\Phi(x) \rightarrow e^{-\sigma_{i}} \Psi(x)
$$

in order to bring the kinetic term to the canonical form. One gets

$$
S_{\Phi, i}=-\int_{B_{i}} d x\left[\frac{1}{2} \eta^{\mu \nu} \partial_{\mu} \Psi \partial_{\nu} \Psi+\frac{1}{2} m^{2} e^{2 \sigma_{i}} \Psi^{2}+\frac{\lambda}{4} \Psi^{4}\right] .
$$

The conclusion, derived on the basis of this formula, states that the mass of the field in the effective theory on brane 2 is $\tilde{m}=m e^{-k R}$. With $m \sim M_{P l}$ and $k R \approx 30 \div 35$ the brane mass $\tilde{m} \sim 1 \mathrm{TeV}$, i.e. the TeV-scale is obtained from the Planck scale due to the exponential factor [1].

However, the induced metric on brane 2, Eq. (34), contains the exponential factor $e^{2 \sigma_{2}}=e^{-2 k R}$ which is exactly of the same origin as the one discussed above, namely it also comes from the warp factor in the background metric, Eq. (4). The coordinates $\left\{x^{\mu}\right\}$ therefore are not Galilean on this brane. As we already mentioned in the Introduction, coordinates are called Galilean if the metric tensor is $\operatorname{diag}(-1,1,1,1)$, see Ref. [10].

In Ref. [11] the correct physical mass of a brane field was determined from the asymptotic behavior of the 2-point function expressed in terms of the proper distance. Here we show that the correct interpretation of the 
effective theory on brane 2 and correct determination of the mass can be achieved simply by changing to the Galilean coordinates

$$
x^{\mu} \rightarrow z^{\mu}=e^{-k R} x^{\mu} .
$$

In these coordinates the action $S_{\Phi, 2}$, Eq. (35), is equal to

$$
S_{\Phi, 2}=-\int_{B_{2}} d z\left[\frac{1}{2} \eta^{\mu \nu} \frac{\partial}{\partial z^{\mu}} \tilde{\Phi} \frac{\partial}{\partial z^{\nu}} \tilde{\Phi}+\frac{m^{2}}{2} \tilde{\Phi}^{2}+\frac{\lambda}{4} \tilde{\Phi}^{4}\right]
$$

where $\tilde{\Phi}(z)=\Phi(x)$. Therefore, the brane field on brane 2 has the physical mass equal to its Lagrangian mass $m$. In fact, expression (37) is very easy to understand. Indeed, since Eq. (33) is covariant, by changing the coordinates $\left\{x^{\mu}\right\}$ to $\left\{z^{\mu}\right\}$ in it we arrive immediately at Eq. (37).

The general form of the interaction of the five-dimensional gravity with matter is given by the following standard expression:

$$
\frac{\hat{\kappa}}{2} \int_{B_{1}} h^{\mu \nu}(x, 0) T_{\mu \nu}^{(1)} d x+\frac{\hat{\kappa}}{2} \int_{B_{2}} h^{\mu \nu}(x, R) T_{\mu \nu}^{(2)} \sqrt{-\operatorname{det} \gamma_{\mu \nu}(R)} d x
$$

where $T_{\mu \nu}^{(1)}$ and $T_{\mu \nu}^{(2)}$ are the energy-momentum tensors of the matter on brane 1 and brane 2 respectively. Substituting (19) into (38), decomposing $b_{\mu \nu}(x, y)$ according to (27) and rescaling the field $\phi$ as in Eq. (26), we find that the interaction on brane 1 is given by

$$
\frac{1}{2} \int_{B_{1}}\left[\kappa_{1} b_{\mu \nu}^{(0)}(x) T^{(1) \mu \nu}+\kappa_{2} \sum_{n=1}^{\infty} \omega_{n}^{(1)} b_{\mu \nu}^{(n)}(x) T^{(1) \mu \nu}-\frac{\kappa_{2}}{\sqrt{3}} \varphi T_{\mu}^{(1) \mu}\right] d x .
$$

The coupling constants of the zero mode, the radion and the massive KK modes on brane 1 are equal to $\kappa_{1}, \kappa_{2} \omega_{n}^{(1)}$ and $\left(-\kappa_{2} / \sqrt{3}\right)$ respectively, where

$$
\kappa_{1}=\hat{\kappa} N_{0} \sim \frac{\sqrt{k}}{M^{3 / 2}}, \quad \kappa_{2}=\kappa_{1} e^{-k R}, \quad \omega_{n}^{(1)}=\frac{\psi_{n}(0)}{N_{0}} e^{k R}
$$

Here we used relations (30), which also imply that $\omega_{n}^{(1)} \sim 1$ for small $n$. In particular, $\omega_{n}^{(1)}=2.48,3.34,4.00,4.58 \ldots$ for $n=1,2,3,4, \ldots$.

Now let us analyze the interaction of the five-dimensional gravity with matter on brane 2. Again, to have the correct interpretation of the effective 
theory on brane 2 we change the coordinates $\left\{x^{\mu}\right\}$ for the Galilean coordinates $\left\{z^{\mu}\right\}$, Eq. (36). In these coordinates the relevant term in Eq. (38) takes the form

$$
\frac{\hat{\kappa}}{2} \int_{B_{2}} h_{\mu \nu}^{\prime}(z, R) T_{\rho \sigma}^{\prime(2)} \eta^{\mu \rho} \eta^{\nu \sigma} d z
$$

where $T^{\prime(2), \mu \nu}$ is the canonical energy-momentum tensor of the matter,

$$
h_{\mu \nu}^{\prime}(z, R)=e^{2 k R} h_{\mu \nu}(x, R),
$$

and the prime labels tensors in the coordinates $\left\{z^{\mu}\right\}$. From now on the fourdimensional indices will be raised and lowered using the Minkowski metric tensor $\eta_{\mu \nu}$.

To get the correct couplings we interprete action (32) as the effective action of the bulk graviton fields and radion on brane 2. In Galilean coordinates (36) it becomes

$$
\begin{aligned}
S_{e f f} & =\int\left[-\frac{1}{4} \sum_{n=0}^{\infty}\left(e^{-2 k R} \frac{\partial}{\partial z^{\mu}} b_{\rho \sigma}^{\prime(n)} \frac{\partial}{\partial z_{\mu}} b^{(n), \rho \sigma}+m_{n}^{2} b_{\rho \sigma}^{(n)} b^{(n), \rho \sigma}\right)\right. \\
& \left.-\frac{1}{2} e^{2 k R} \frac{\partial}{\partial z^{\mu}} \varphi^{\prime} \frac{\partial}{\partial z_{\mu}} \varphi^{\prime}\right] d z .
\end{aligned}
$$

Rescaling the graviton field and the radion field as

$$
b_{\rho \sigma}^{(n)}(z)=e^{k R} u_{\rho \sigma}^{(n)}(z), \quad \varphi^{\prime}(z)=e^{-k R} \chi(z)
$$

respectively, we obtain the effective action in the canonical form

$$
\begin{aligned}
S_{e f f} & =\int\left[-\frac{1}{4} \sum_{n=0}^{\infty}\left(\frac{\partial}{\partial z^{\mu}} u_{\rho \sigma}^{(n)} \frac{\partial}{\partial z_{\mu}} u^{(n) \rho \sigma}+\left(m_{n} e^{k R}\right)^{2} u_{\rho \sigma}^{(n)} u^{(n) \rho \sigma}\right)\right. \\
& \left.-\frac{1}{2} \frac{\partial}{\partial z^{\mu}} \chi \frac{\partial}{\partial z_{\mu}} \chi\right] d z .
\end{aligned}
$$

We see that the masses of the gravitons as measured by an observer on brane 2 are equal to

$$
M_{n}=m_{n} e^{k R}=k \beta_{n} .
$$

Contrary to the case of brane fields described by action (33), the graviton mass spectrum is multiplied by the scale factor $e^{k R}$ when we pass from the 
coordinates $\left\{x^{\mu}\right\}$ to $\left\{z^{\mu}\right\}$. The reason is that expression (32) is not covariant, namely the indices are raised with the Minkowski metric whereas the metric in the coordinates $\left\{x^{\mu}\right\}$ is $\gamma_{\mu \nu}(x, R)=e^{-2 k R} \eta_{\mu \nu}$.

We would like to note that in fact relation (44) between the Lagrangian mass and the mass measured by an observer on brane 2 is valid for bulk fields of any tensor structure (see an example in [11]).

Using Eq. (19) and making the mode decomposition accompanied by rescaling (42) interaction term (41) on brane 2 takes the form

$$
\begin{aligned}
& \frac{1}{2} \int_{B_{2}}\left[\hat{\kappa} e^{k R} \psi_{0}(R) u_{\mu \nu}^{(0)}(z) T^{(2) \mu \nu}+\hat{\kappa} \sum_{n=1}^{\infty} e^{k R} \psi_{n}(R) u_{\mu \nu}^{(n)}(z) T^{(2) \mu \nu}\right. \\
& \left.-\frac{\hat{\kappa}}{\sqrt{3}} \frac{\sqrt{k}}{\sqrt{1-e^{-2 k R}}} \chi T_{\mu}^{(2) \mu}\right] d z \\
& =\frac{1}{2} \int_{B_{2}}\left[\kappa_{2} u_{\mu \nu}^{(0)}(z) T^{(2) \mu \nu}-\kappa_{1} \sum_{n=1}^{\infty} \omega_{n}^{(2)} u_{\mu \nu}^{(n)}(z) T^{(2) \mu \nu}\right. \\
& \left.-\frac{\kappa_{1}}{\sqrt{3}} \chi T_{\mu}^{(2) \mu}\right] d z
\end{aligned}
$$

The couplings of the zero mode, massive KK modes and the radion are $\kappa_{2}$, $\left(-\kappa_{1} w_{n}^{(2)}\right)$ and $\left(-\kappa_{1} / \sqrt{3}\right)$ respectively, where $\kappa_{1}$ and $\kappa_{2}$ were defined by Eq. (40) and

$$
\omega_{n}^{(2)}=-\frac{\psi_{n}(R) e^{k R}}{N_{0}}
$$

Using Eqs. (31) it can be easily checked that $\omega_{n}^{(2)} \approx 1.0$ for small $n$.

We see that the order of magnitude of the graviton and radion interactions with matter on both branes are set by two parameters. We have chosen them to be $\kappa_{1}$ and $\kappa_{2}$, the couplings of the massless graviton to mater on brane 1 and brane 2 respectively. They differ in the exponential factor $e^{-k R}$ (see Eq. $(40))$.

Let us discuss the effective models on brane 1 and brane 2 . In the conventional and physically interesting scenario it is assumed that our brane is brane 2, whereas brane 1 accommodates a mirror world. To have usual Newton's gravity mediated by the massless graviton we identify its coupling constant $\kappa_{2}$ with $1 / M_{P l}$. Combining Eqs. (29) and (40) we obtain the following relation between the Planck mass and the fundamental mass scale $M$ 
(recall that $\left.\hat{\kappa}^{2}=M^{-3}\right)$ :

$$
M_{P l}^{2}=\frac{M^{3}}{k}\left(e^{2 k R}-1\right) \approx \frac{M^{3}}{k} e^{2 k R} .
$$

By choosing $M \sim k \sim 1 \mathrm{TeV}$ we reproduce the correct value of the Planck mass, if the argument of the exponential factor satisfies $k R \approx 30 \div 35$. From Eqs. (40), (46) it follows that, as in the standard scenario, the couplings of the massive KK modes and of the massless radion to matter on brane 2 are of order of $1 \mathrm{TeV}^{-1}[6,7]$. The effective Lagrangian (46) can be rewritten in the following form, from which the above features are clearly seen:

$$
\frac{1}{2} \int_{B_{2}}\left[\frac{1}{M_{P l}} u_{\mu \nu}^{(0)}(z) T^{(2) \mu \nu}+\sum_{n=1}^{\infty} \frac{\omega_{n}^{(2)}}{\Lambda_{\pi}} u_{\mu \nu}^{(n)}(z) T^{(2) \mu \nu}-\frac{1}{\Lambda_{\pi} \sqrt{3}} \chi T_{\mu}^{(2) \mu}\right] d z,
$$

where $\Lambda_{\pi}=M_{P l} e^{-k R}=\sqrt{M^{3} / k} \sim 1 \mathrm{TeV}$ and $M_{P l}$ is given by (47). The masses of the KK excitations are given by Eq. (44). For small $n$ the masses $M_{n} \sim k \sim 1 \mathrm{TeV}$. The presence of the massless radion with such coupling leads to some predictions, which are in contradiction with the available high energy physics data. To avoid this problem several mechanisms for generating a mass for the radion were proposed (see, for example, Ref. [21]).

Now we turn to the analysis of the positive tension mirror brane (brane 1 ). The coupling $\kappa_{1}$ of the massless graviton is related to the fundamental mass scale $M$ and the parameter $k$ as

$$
\kappa_{1}^{-2}=\frac{M^{3}}{k}\left(1-e^{-2 k R}\right)
$$

With the parameters defined by physical conditions imposed on brane 2 the coupling of the massless graviton $\kappa_{1} \sim 1 / \Lambda_{\pi}$, whereas the couplings of the massive gravitons and of the radion to matter turn out to be $\sim 1 / M_{P l}$.

Suppose for a moment that brane 1 is our brane. In this case we would have to require that $\kappa_{1} \sim M_{P l}^{-1}$. This can be achieved by taking $M \sim k \sim M_{P l}$ and keeping $\exp (k R) \gg 1$. Then, from relations (40), we would get that $\kappa_{1} \gg \kappa_{2}$, i.e. the interactions of the massive KK modes and of the radion field with matter on brane 1 would be much weaker (exponentially suppressed) than that of the massless graviton. Therefore, on brane 1 the radion field would not affect Newton's gravity, which is determined by the interaction of the massless graviton in (39). The massive KK modes have masses of the order of $M_{P l} e^{-k R}$. Thus, such scenario would be phenomenologically acceptable, but not interesting. 


\section{Conclusions and discussion}

We presented a consistent Lagrangian description of linearized gravity in the Randall-Sundrum model with two branes paying attention to some issues which were skipped or received little attention in previous publications. In particular, we discussed the gauge freedom and gauge fixing, and carried out the diagonalization of the Lagrangian and decoupling of the classical equations of motion in detail. We also revised the mode expansion of the bulk gravitational field. These enabled us to identify the physical degrees of freedom in the RS1 model and construct the effective Lagrangian. We would like to note that in the limit $R \rightarrow \infty$ the radion field $\phi$ drops from the Lagrangian, and we get the same degrees of freedom, as found in [17] for the case of infinite extra dimension.

An important point in our studies is that the five-dimensional gravity looks different when viewed from different branes. This point was stressed and worked out in some papers, see, for example, $[4,11]$. Our central observation is that in order to have the correct interpretation of the effective theories on the branes and to calculate the values of masses and coupling constants measured by a brane observer one has to change to the proper (Galilean) coordinates. We showed that contrary to the conventional interpretation the measured masses of brane fields coincide with the Lagrangian mass (see Eq. (37)). The masses of bulk fields measured on the brane at $y=R$ acquire the enhancing exponential factor comparing to the Lagrangian masses, see Eq. (44). As we have mentioned in the Introduction, in Ref. [11] these mass relations were obtained from the analysis of the 2-point Green functions. We also derived the relation between the Planck mass and the fundamental scale of the five-dimensional gravity, Eq. (47), which differs from the conventional one.

As a matter of fact, instead of choosing the Galiliean coordinates for description of the effective theory on brane 2 only, one can introduce global five-dimensional coordinates $\left\{z^{\mu}, y\right\}$, for which the warp factor on brane 2 is equal to 1, i.e. $\left\{z^{\mu}\right\}$ are Galilean coordinates on brane 2. This alternative description was used in Ref. [4] for derivation of relation (47). All conclusions concerning the physical content of theories on brane 1 and brane 2, obtained there, coincide with ours.

Our expression for the Lagrangian of the effective four-dimensional theory on brane 2 in the physical (Galilean) coordinates, given by (43), (46), differs 
from the one usually discussed in the literature (see, for example, Refs. $[6,7]$ ). In particular, we get $M \sim k \sim 1 \mathrm{TeV}$, whereas in the above mentioned papers one needs $M \sim k \sim M_{P l}$. In our case, according to Eq. (44) the spacings between masses in the spectrum are $\sim k \sim R^{-1}$, similar to the usual KaluzaKlein theories with direct product topology of the space-time. If the analysis of the RS model is carried out in non-Galilean coordinates, as it is done in many papers, then the spacings are $\sim k e^{-k R} \sim R^{-1} e^{-k R} \ll R^{-1}$, which is valid only on brane 1 .

Essentially, the hierarchy, i.e. the existence of two scales separated by many orders of magnitude, is generated in the same way as in the conventional interpretation. Namely, according to Eq. (47) the scale of fivedimensional theory turns out to be exponentially suppressed with respect to the scale of four-dimensional gravity on brane 2 . In fact this possibility was mentioned briefly in Ref. [2] (see also [4]). As it can be seen from the first term in Eq. (45), the exponential factor comes from $e^{k R} \psi_{0}(R) \sim \sqrt{k} e^{-k R}$, i.e. from the exponential decay of the graviton wave function which is proportional to the warp factor in the background metric.

The relative strengths of interaction of massless and massive gravitons with matter are explained by different rates of the falloff of the wavefunctions of these fields along the fifth direction. Indeed, from Eqs. (45), (46) it follows that

$$
\frac{\kappa_{2}}{\kappa_{1} \omega_{n}}=-\frac{\psi_{0}(R)}{\psi_{n}(R)} \sim e^{-k R} .
$$

One last remark. Though formulas (40) and (47), expressing the couplings $\kappa_{1}$ and $\kappa_{2}$ in terms of the parameters $M, k$ and $R$ of the model, are different from the ones used in the literature (see $[1,6,7]$ ), it is easy to check that the ratios $\kappa_{1} / \kappa_{2}$ and $\kappa_{1} \omega_{n}^{(2)} / \kappa_{2}$ are the same both in physical (Galilean) coordinates $\left\{z^{\mu}\right\}$ and in "non-physical" coordinates $\left\{x^{\mu}\right\}$. For this reason phenomenological predictions obtained in previous papers remain valid.

\section{Acknowledgments}

The authors are grateful to G.Yu. Bogoslovsky, Yu.V. Grats and V.A. Rubakov for useful discussions. The work of E.B., Yu.K. and I.V. was supported by RFBR grant 00-01-00704 and the grant 990588 of the programme "Universities of Russia". E.B. was supported in part by the CERN-INTAS grant 99-0377 and by the INTAS grant 01-0679. Yu.K. and I.V. were also supported in part by the programme SCOPES (Scientific co-operation between 
Eastern Europe and Switzerland) of the Swiss National Science Foundation (project No. 7SUPJ062239) and financed by the Swiss Federal Department of Foreign Affairs.

\section{References}

[1] L. Randall and R. Sundrum, Phys. Rev. Lett. 83 (1999) 3370.

[2] L. Randall and R. Sundrum, Phys. Rev. Lett. 83 (1999) 4690.

[3] I. Antoniadis, "String and D-brane Physics at Low Energy", hepth/0102202.

[4] V.A. Rubakov, "Large and infinite extra dimensions: an introduction", hep-ph/0104152.

[5] Yu.A. Kubyshin, "Models with Extra Dimensions and Their Phenomenology", hep-ph/0111027.

[6] H. Davoudiasl, J.H. Hewett and T.G. Rizzo, Phys. Rev. Lett. 84 (2000) 2080 .

[7] H. Davoudiasl, J.H. Hewett and T.G. Rizzo, Phys. Rev. D63 (2001) 075004.

[8] R.-D. Heuer, D.J. Miller, F. Richard, P.M. Zerwas (editors), "TESLA. Technical Design Report. Part III: Physics at an $e^{+} e^{-}$Linear Collider." DESY 2001-011 (March 2001), hep-ph/0106315.

[9] M. Besançon, "Experimental introduction to extra dimensions", hep$\mathrm{ph} / 0106165$.

[10] L.D. Landau and E.M. Lifshitz, "The Classical Theory of Fields". Pergamon Press, Oxford (1975).

[11] B. Grinstein, D.R. Nolte and W. Skiba, Phys. Rev. D63 (2001) 105005.

[12] J. Garriga and T. Tanaka, Phys. Rev. Lett. 84 (2000) 2778.

T. Tanaka and X. Montes, Nucl. Phys. B582 (2000) 259. 
[13] E.E. Boos, Yu.A. Kubyshin, M.N. Smolyakov and I.P. Volobuev, hepth/0105304.

[14] B.S. DeWitt, "Dynamical Theory of Groups and Fields", Gordon and Breach, New York, 1965.

[15] L.P. Grishchuk, A.N. Petrov and A.D. Popova, Comm. Math. Phys. 94 (1984) 379.

[16] Ch. Charmousis, R. Gregory, V. Rubakov, Phys. Rev. D62 (2000) 067505 .

[17] I.Ya. Aref'eva, M.G. Ivanov, W. Mück, K.S. Viswanathan, and I.V. Volovich, Nucl.Phys. B590 (2000) 273.

[18] N. Arkani-Hamed, S. Dimopoulos and J. March-Russel, Phys. Rev. D63 (2001) 064020.

[19] R. Sundrum, Phys. Rev. D59 (1999) 085010.

[20] C. Csáki, M. Graesser, L. Randall and J. Terning, Phys. Rev. D62 (2000) 045015.

W.D. Goldberger and M.B. Wise, Phys. Lett. B475 (2000) 275.

[21] W.D. Goldberger and M.B. Wise, Phys. Rev. Lett. 83 (1999) 4922. 